\title{
Design and performance evaluation of cost-effective function-distributed mobility management scheme for software-defined smart factory networking
}

\author{
Jeong-A. Kim ${ }^{1}$. Do Gun Park ${ }^{1}$ Jongpil Jeong ${ }^{1}$
}

Received: 2 January 2019 / Accepted: 5 May 2019 / Published online: 14 June 2019

(c) The Author(s) 2019

\begin{abstract}
With the proliferation of mobile devices, Internet of Things (IoT), and edge commuting, and the advancement of ICT, mobile computing has become the norm, rather than the exception. Current network technologies and distributed system architectures make it difficult to meet users' network trace, and incur high processing costs. To solve this problem, this paper proposes new functional distributed mobility management technologies for the application of the industrial future Internet. This implies the need for an efficient distributed mobility system to apply 5G or TSN (time sensitive network) technologies to the future industry Internet. Thus, it is assumed that various analytical techniques will be applied to provide superior performance in cost analyses, such as location update costs and packet transport costs. In this paper, we propose a cost-effective functiondistribution mobility management system that will build a future industrial Internet to meet the needs and convenience of users. It also uses computer simulation to create the mobility provision system, and the electiveness of the cost analysis usage and rental management function models. Software-defined networking in smart factories (SFs) enables them to easily adapt the communication network to changing requirements. Similar to cloud-based systems, such SFs could be seen as producing clusters that could be rented and configured as needed. To achieve the required flexibility, the SF network uses software defined networking combined with network function virtualization. Despite the fact that the technology is not yet ready for deployment in today's manufacturing networks, a novel network architecture for SFs based on software-defined networking and network virtualization is here proposed, to support smart services, especially for Industry 4.0.
\end{abstract}

Keywords Function-distributed mobility management $\cdot$ Future industrial internet $\cdot$ Proxy mobile IP $\cdot$ Software-defined networks $\cdot$ Smart factory networking

\section{Introduction}

The increasing number of applications and smart services on the go in every domain and the widespread adoption of these services pose new challenges to the supporting infrastructure related to networking performance, scalability, architecture sustainability, user and data privacy, security, and service

Jongpil Jeong

jpjeong@skku.edu

Jeong-A. Kim

jkop109@skku.edu

Do Gun Park

pdgun@skku.edu

1 Sungkyunkwan University, 2066 Seobu-ro, Jangan-gu, Suwon, Gyeonggi-do 16419, Korea delivery, among others. In addition, the enormous amount of data collection that takes place in these services/applications is opening incredible opportunities, yet presents a plethora of challenges for sensing, transmission, storage, processing, management, and analytic. Such increased complexities at the different levels of the entire software and hardware stack require new paradigms, architectures, and protocols to tackle dynamic topology in mobile networking, resource constraints, heterogeneous architectures, the impracticality of centralized approaches, weak survivability, and unattended resolution of potential failures. Novel contributions in machine learning techniques and big data analytic frameworks are also required to turn big sensor data into actions, embed intelligence into service operation, provide deep insights, generate predictive analytic, and support real-time decision-making. Accordingly, researchers are conducting new future network research in a variety of 
ways to address user needs. Future network technologies have recently evolved toward 5G (Condoluci and Mahmoodi 2018; Frias and Martínez 2018).

Traditional network technologies can no longer meet these requirements, because of problems caused by new mobile devices, communications services, and network traffic (Cheng et al. 2018). It has found its way into our daily life, be it private or in business, e.g., through social networks, IP telephony, video streaming, cloud applications, and the like. Along these lines, it can be envisioned that, in the near future, almost everything will be networked, and connected to and by the Internet. This way, the Internet of Things will more and more become reality. The digital and analog worlds will become increasingly interwoven, and so-called smart environments will emerge, which promise to improve our quality of life, and enable more economic usage of our resources. Emerging smart factories (SFs) are envisioned to be seamlessly integrated into such smart environments of the future (Rong et al. 2016; Boulaalam 2016; Darwish and Hassanien 2018). Consequently, production, networking, and computing will become tightly integrated. Cooperation among different sets of a factory, or between different factories, will be easily possible. It is envisioned that SFs will be highly modularized, and to a large extent driven by IT services. For example, it is expected that they will be largely self-organizing, to efficiently adapt themselves to frequently changing requirements. The resulting possibilities for flexible and timely manufacturing have the potential for innovation, and may lead to notable changes in manufacturing practices (Bormann et al. 2012). To achieve the aforementioned objectives, future SFs require, among other things, i.e., they should be easily recognizable, and it should be possible to treat data streams in the network differently, according to their individual requirements. Further requirements include an easy way to manage the network, as well as high robustness and appropriate IT security considerations (Moritz et al. 2013). Due to the integration of various wireless terminals, there is increasing demand for seamless services anytime, anywhere, and on the move. Thus, various mobility-related protocols have been suggested to enable services not only within a specific network, but also among multiple protocols, backbones, different devices, and Internet service providers.

To solve this problem, the $5 \mathrm{G}$ network and time sensitive networking (TSN) technology were recently developed. 5G can provide speeds above $4 \mathrm{G}$, and compared to the previous generation of communication technologies, $5 \mathrm{G}$ communication networks have more than 1000 times increased communication capacity. They also gain tremendous improvements from the (10 to 100) times increase of data transfer rates. $5 \mathrm{G}$ can extend the benefits of mobile technology to connect, control, exchange, search and link all people, and prepare for spatial and temporal limitations (Oughton and Frias 2017). 5G connects various smart devices to the Internet, and has characteristics such as automation, smart links, and real-time monitoring (Alejandro et al. 2018). It is currently planned to apply this technology to the industrial Internet industries' Internet of Things (IoT). TSN technology is based on Ethernet to provide low-latency, low-latency, and low-loss services (Prinz et al. 2018). This synchronizes the time between components that share network resources, and allows traffic to be handled through scheduling based on the time that is synchronized. This ensures maximum delay in switching within the equipment, and allows efficient operation of the equipment. The reality is that there are not enough solutions to solve problems, such as high capacity, variety of equipment, stable reliability, and mobility. In this paper, it is thought that future Internet, such as 5G or TSN, will be proposed to enhance user mobility, thereby improving satisfaction with user requirements, and applying them to industrial sites.

The MIP (Mobile IP) (Alotaibi et al. 2018) in the current IP network is a standard movement-supporting protocol. Since this MIP (Johnson et al. 2004) was used, a few similar mobile protocols have been suggested to be able to handle more effectively specific communication goals which are appropriate to demands of users. The fast mobile IP (FMIP) (Koodli 2009) of these is a protocol to carry out the fast handover using the context transmission technology. The hierarchical mobile IP (HMIP) (Soliman et al. 2008) has an effect on the reduction of the early communication signal cost by using the local binding update procedure of the layer network composition. The proxy mobile IP (PMIP) (Gundavelli et al. 2018) transplants the mobile function in the edge node (intersection point of the communication network and $\mathrm{MN}$ access point), and accordingly it is appropriate to reduce the workload of the MN. The network layer structure and the centralized management system are generally used in the mobility management of mobile communication networks. The next generation mobile networks, such as system architecture evolution/evolved packet core (SAE/ EPC) and long term evolution (LTE) are considered at the general packet radio system (GPRS)-based and IP-based mobile management system, and are standardized for every IP mobile network (Copel and Crespi 2011; Magnano et al. 2015). The PMIP is adopted as the IP-based mobile protocol. As the Internet technology develops in the future, IP mobility and mobile phone mobility are generally harmonized and integrated.

However, recently it is required to provide easy cooperation between sites within factories, and with other factories. In other words, in SFs, communication is required between the factory floor and manufacturing zone, as well as connectivity towards office IT, or remote production facilities that are connected via wide area network or Internet. As the ideas of SFs are being constructed, which closely integrate 
production, networking, and computing, the method for supporting global mobility from the perspectives of intellectualization and SF is reinterpreted. Through numerous Internet research works and studies, major functions, such as the security of future Internet, contents transmission mechanism, delay-tolerant network, frame management and control, service technology, routing, and future Internet infra planning of experiments, are being standardized. The mobility between them is one of the important characteristics in the technology of management, framework and transmission path, etc. MME (Jin et al. 2010) appears to be one of the promising technologies that are related to mobility problems, such as multi-homing and ID/location (radio locator) distribution. This paper proposes a new mobility management framework. The suggested mobility management framework provides an effective technique to construct flexible systems, and accordingly could be easily applied to the future Internet. In terms of the cost of location update and packet analysis, this suggested technique shows analysis results of better performance.

Software-defined networks and network virtualization provide a promising basis for flexible requirement-driven network configuration and operation. These are currently heavily discussed trends on the Internet, but to some extent, they are still in their infancy. However, in dedicated exemplary settings, they have already proven their tremendous potential, e.g., in the context of cloud computing, and regarding the design of modern data centers, wide area networking, or mobile core networks (Gandhi et al. 2014; Hong et al. 2013; Jain et al. 2013; Basta et al. 2014). Industrial networking and upcoming SFs as outlined above could also profit from the higher flexibility provided by SDN. This could lead to tremendous improvements in manufacturing lines and factory floors, and large changes to traditional practices in manufacturing (Girbea et al. 2014; Eliasson et al. 2015). IT services may very well increasingly shape the design of future factories. It is our vision that softwaredefined networks and virtualization are viable approaches that enable flexible and cost-efficient resource sharing within and among SFs, leading to a new quality of manufacturing processes (Konieczek et al. 2016). They have the potential to change industrial networking in a manner similar to the changes that virtualization and cloud computing brought to the Internet.

The composition of this paper is as follows. Section 2 presents the software-defined networking, network virtualization, and mobility management techniques for cost-effective functional delegation. Section 3 describes the cost-effective function-distributed mobility management scheme for software defined smart factory networking, while Sect. 4 discusses the performance evaluation of the mobility management techniques in detail. Finally, Sect. 5 concludes this paper.

\section{Background and related work}

The mobility management scheme proposed in this paper is divided into location management and handoff management for each network device. In general, handoff management is defined as a technique that enables continuous service at the application layer, when the MN moves between domains or cells. A major advantage of SDN is its increased flexibility, compared with traditional network designs.

\subsection{Software-defined networking}

The main concept behind SDN is a consequent decoupling of the control and data planes, as shown in Fig. 1.

Following the definition of the Open Networking Foundation, an architecture for SDN can be subdivided into three distinct layers application plane, control plane, and data plane (Foundation 2013). The application plane consists of software programs that make use of a northbound interface exposed by an SDN controller to explicitly program the behavior of the network. Such programs (SDN applications) are responsible for tasks like routing, access control, load balancing, or network isolation. In fact, any kind of network control functionality can be implemented as a dedicated SDN application. The application developer is only restricted by the functionality provided by the northbound interface, and not, for example, by the innovation cycle of a hardware vendor. The control plane is represented by a logically centralized SDN controller, which can best be described as an operating system for SDN applications. Its main task is to establish and maintain an abstract global view of the current network, and provide this view to SDN applications via the northbound interface. It is also responsible for translating the input of SDN applications (i.e., commands received via the northbound interface)

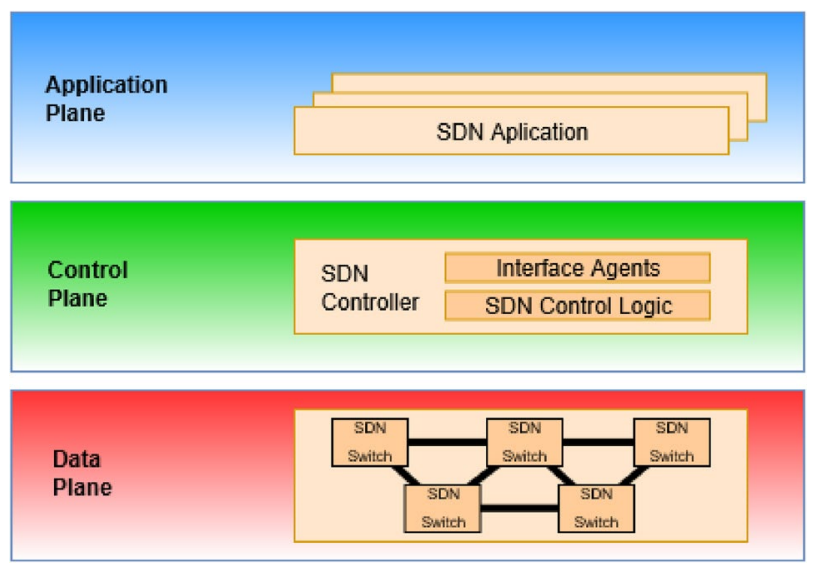

Fig. 1 Software-defined networking (SDN) layer 
into instructions that can be understood by switches inside the physical infrastructure. The SDN controller runs on a powerful server, and thus provides higher flexibility for executing complex control tasks, compared with switches or routers that are typically limited with respect to computational power and overall memory size. Note that while the control plane is logically centralized, the actual deployment inside the physical infrastructure can be distributed to realize scalability and robustness (e.g., by using a federation of multiple controllers). Finally, the data plane comprises SDN-enabled switches that expose their capabilities to the SDN controller using a standardized, open, and vendor neutral control data plane interface. For simplicity, the latter is often termed a southbound interface. The Open Networking Foundation defines the following tasks that are associated with the southbound interface: programmatic control of all forwarding operations, reporting of switch capabilities and statistics, and event notification For simplicity, the latter is often termed a southbound interface. Control of forwarding operations in the data plane is realized by rules that are remotely installed into SDN switches by the SDN controller on behalf of SDN applications. Every rule consists of a set of match fields, and a set of actions. The match fields can be seen as a filter function identifying all packets that belong to a specific flow, and the corresponding actions determine which commands are executed for all packets of that flow. Well-known southbound interfaces like Open Flow (Hu et al. 2014) provide packet header matching capabilities for many common Internet protocols (IP/MAC source and destination addresses, TTL, DSCP, VLAN tag, and so on), and various actions, like sending a packet towards a specific egress port, performing header modifications, or enabling differentiated packet treatment to support Quality of Service. If an SDN switch has no match fields for an incoming packet, that packet is dropped, or forwarded towards the SDN controller to decide on further treatment of the associated packet flow (e.g., its individual route through the network). Within the traditional Internet-based networking architectures, the routers are responsible for both control plane (i.e., executing routing protocols) and data plane operations (i.e., performing destination based packet forwarding) (Sezer et al. 2013). They use distributed protocols in the control plane that are implemented by the vendor directly within the router hardware. A drawback is the standardized and rugged functionality, e.g., always routing along shortest paths, thereby not allowing more flexible routing policies. Moreover, the distributed approach sometimes makes system behavior less predictable and deterministic. On the other hand, with the SDN, all high level network control operations, like routing, are handled by the SDN controller and the SDN applications, while the hardware inside the data plane is only responsible for the actual packet treatment (e.g., on switch x forward packet up to switch y using interface $\mathrm{z}$ ). A main advantage is that this approach can use more sophisticated and individual forwarding strategies (e.g., time-dependent routing) implemented in software. In addition, SDN provides network operators with easier and more efficient automated network management, due to open interfaces for remote programmability and fine grained centralized network control. Because new functionality can be introduced by simply changing the applications, SDN also enables rapid prototyping and high flexibility, e.g., by deploying applications that provide traffic engineering, monitoring, or support for certain aspects of security and dependability (Kreutz et al. 2015). A potential drawback of SDN is its limited scalability with respect to the number of individual flows, which might result in a high number of control messages between SDN switches and controller, or the limited number of flow forwarding entries supported by hardware switches. However, the expected number of flows within an SF networking environment seems not to be critical with respect to scalability limits.

\subsection{Network virtualization}

In addition to SDN, the trend toward network virtualization also bears great potential to revolutionize the provisioning of networked applications in general. In the context of this paper, we focus on NFV. The core idea behind NFV is the virtualization of network services, e.g., firewalls, gateways, and related functionality, which are normally realized using dedicated hardware systems inside the data path (also called middle boxes). With NFV, such services can be realized software-based inside a virtual machine, and provisioned on demand, thereby enabling high flexibility, similar to the resource pooling principle known from cloud computing. To use this flexibility, additional components are required inside the software-defined network. First, an NFV infrastructure is needed where the virtualized network services can be deployed. This might be an edge cloud or a set of dedicated servers (commodity hardware) directly collocated with the physical networking infrastructure. Second, the control plane must be extended to support NFV orchestration, to handle placement and life cycle management of the virtual machines. Network functions virtualization provides advantages with respect to resource scalability and resilience, since the number of NFV instances can be scaled up and down, as required by the current situation. In particular, the functionality provided by virtualized services is no longer tied to a fixed position within the network topology, which greatly enhances the configurability of softwaredefined networks.

Figure 2 shows an architectural sketch of a network with explicit support for SDN and NFV. A joint control plane that consists of an SDN controller and an NFV orchestrator is responsible for controlling the network, configuring network services, and managing NFV instances. If a specific 
Fig. 2 Architecture of a software-based network with support for network virtualization. $N F V$ network functions virtualization, $S D N$ softwaredefined networking

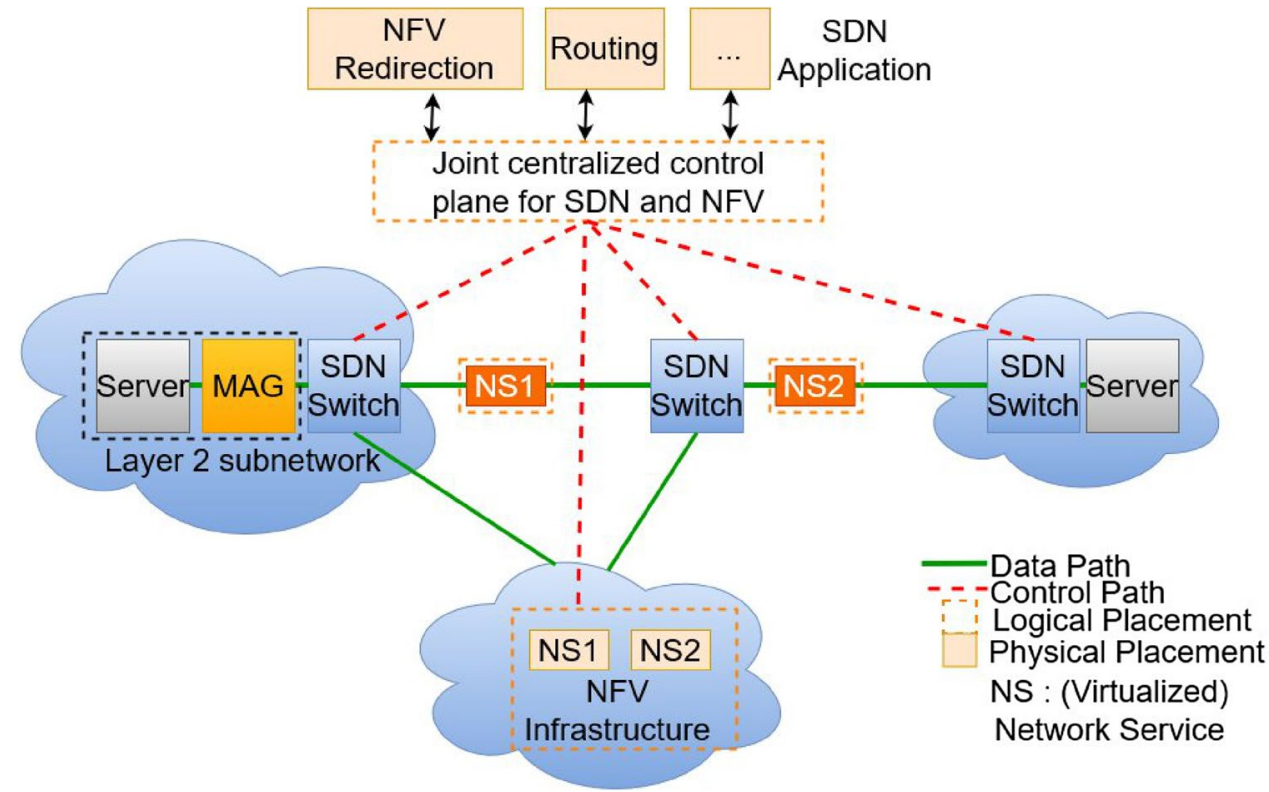

network service is requested, e.g., NS1 in Fig. 2, a new virtual machine with the required functionality is spawned on a compute node inside the NFV infrastructure. Note that the logical placement of the service and the physical placement of the virtual machine can be controlled separately from each other. An SDN application inside the control plane then subsequently directs some or all of the flows in the network, so that they have to pass the new virtual machine, before they can reach their original target (NFV redirection). If multiple network services are associated with one flow, service chaining in conjunction with packet attached metadata (so called network service headers) may be used to enforce all packets of a flow having to pass through a specific set of network services.

\subsection{Mobility management in PMIPv6}

PMIPv6 has increased resource usage in the wireless section, due to signaling between the mobile node (MN) and the access router. In addition, there are limitations in mobility support due to limited performance and resources. As a result of this problem, a network-based protocol has been proposed. PMIPv6 consists of new components of local mobility anchor (LMA), mobile access gateway (MAG), and authentication, authorization, and accounting (AAA) server (Modares et al. 2016). The LMA acts as a kind of home agent for the mobile node in the domain. MAG is mainly located in the access router (AR). Therefore, the MAG becomes the first hop to which the mobile node directly connects, and performs signaling on behalf of the mobile node. The mobile node performs the L2 access authentication, and notifies the MN to the MAG in this process. MAG performs the authentication process with AAA.
The MAG sends a PBU message to the LMA that is responsible for the mobile node to update its current location. Upon receiving this $\mathrm{PBU}$, the $\mathrm{LMA}$ will send a $\mathrm{PBA}$ message to the MAG for the service of the terminal. Then, the LMA creates a bidirectional tunnel between LMA and the MAG using the address of MAG, and prepares the service. When the connection setting is completed, the MAG transmits all traffic from the mobile node to the LMA using the tunnel connected with the LMA. On the other hand, the LMA transmits all the traffic from the outside to the MAG that manages the MN. By performing such communication, the burden on the $\mathrm{MN}$ is reduced. After that, by simplifying the registration procedure of the $\mathrm{MN}$ relating to the movement, it is possible to reduce the communication cost. Figure 3 shows an overview of the explanation.

\subsection{Centralized mobility management method}

For mobility management systems using geographic fixed anchors, user mobility support has some weaknesses in terms of signal cost and data transfer cost. Therefore, the dynamic control mobility management system is being analyzed and studied from various aspects. Chen et al. (2002) and Choi and Tekinay (2002) studied dynamic location update technique from the viewpoint of location information update cost and paging cost analysis. Ghosh and Ghosh (2019) provides trajectory-indexing to efficiently handle huge real-time trajectory updates, geotagging, map-matching services in a distributed computing platform and trajectory-processing based spatio-temporal queries using Traj-Cloud. Generally, the central mobility management system is considered a basic concept. However, centrally managed nodes have disadvantages in 
Fig. 3 Basic structure of PMIPv6

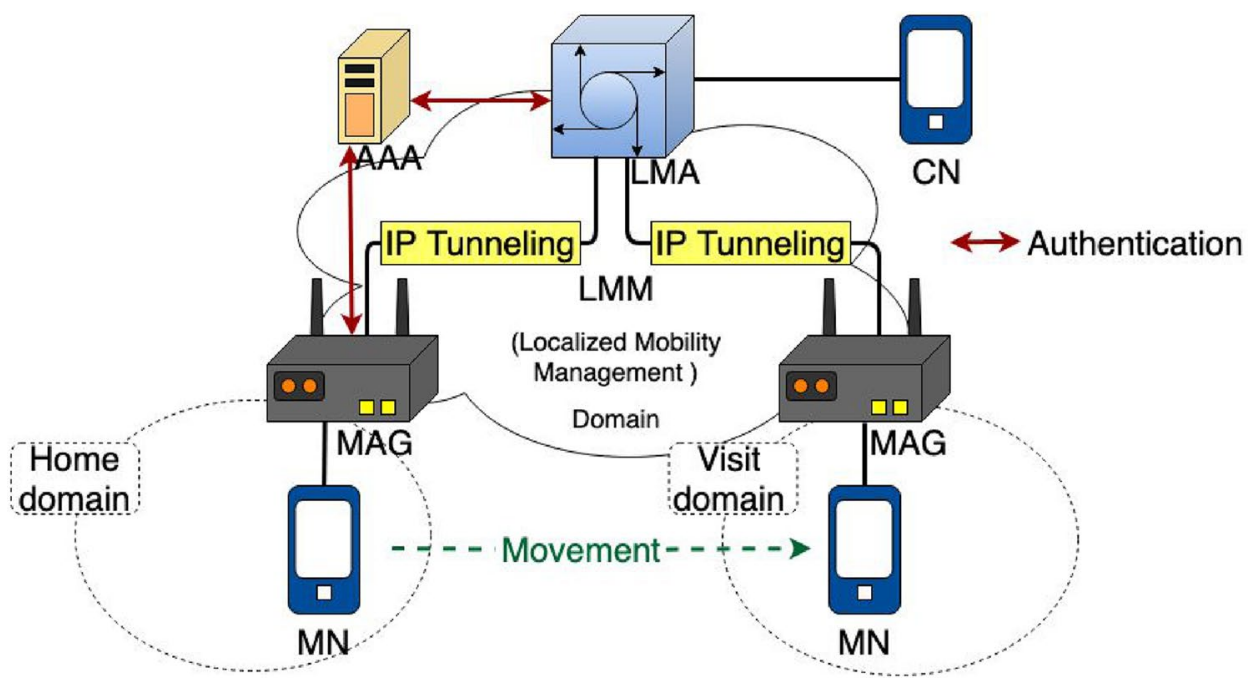

a single point, which is an obstacle to the construction of a flexible system. Therefore, the distributed management system has been studied from various aspects. Zheng et al. (2002) proposed the temporary home agent (HA) from the visited network. In order to avoid single point failure, dynamic HA was assigned and executed. As a result, signal cost effect was obtained at the same time. Numerous effective mobility methods have been continuously studied. However, most of their geographical locations may be dynamically changed, even considering the main function of concentrated single mobility on the nodes. We are designing a network of mobility-managed nodes composed of several functions, such as location management, trust (authentication) management, and state management. Each function has its own characteristics, and there are appropriate places according to those characteristics. Single mobility cannot manage all functions simultaneously on one node. It shows inefficiency in terms of system management. We provide a distributed mobility management system that distributes functions concentrated in one place for more efficient management. This makes it possible to satisfy the necessity of each function appropriately, and it becomes possible to build a more flexible and wide mobility management system.

\section{Cost-effective function-distributed mobility management scheme for software-defined smart factory networking}

Here we explain the system to which is applied the mobility management system through the function distribution. Figure 4 shows a diagram of the composition of the mobility factory network. The composition network in office has path and status location information for external reference to every user. It also has a certification function for users in its network. Each factory has the factory control unit (FCU) which is responsible for and controls the inside of the factory, and the function is authorized by M_FCU. Each factory is authorized with the certification function to certify the internal users of each domain and the function which is related to status location information. That is, H_FCU and V_FCU demonstrate the node which is authorized (authorized functions: location and state) by M_FCU.

When the mobile node (MN) attempts to access the visit network (factory 2) by coming out of the home network (factory 1), V_FAG automatically senses the movement of the $\mathrm{MN}$, and forms a normal wireless connection through the V_FCU and AAA_V (certification). The entire traffic between the $\mathrm{MN}$ and the correspondent node $(\mathrm{CN})$ communicate through $\mathrm{V} \_F C U$, and the $\mathrm{CN}$ checks up the information related to location, status and certification through M_FCU and AAA_M, and can communicate with the MN.

In general, the mobility management system manages the entire mobility related functions by all-in-one in the same, central place. As for the centralized management system, if any function of the central system doesn't function properly, all the users cannot receive normal service. Because every function is centralized, the probability of communication disruption could be high. Each function of the management system has its own unique property. In order to carry out the mobility management effectively in the complex and highly-movable Internet environment, each function must be distributively arranged at the appropriate place according to its unique property. So, the function distribution mobility system is suggested as a way to solve this problem. The mechanism of this distribution mobility system is carried out by authorization. A detailed explanation of the proposed scheme is discussed as follows. 


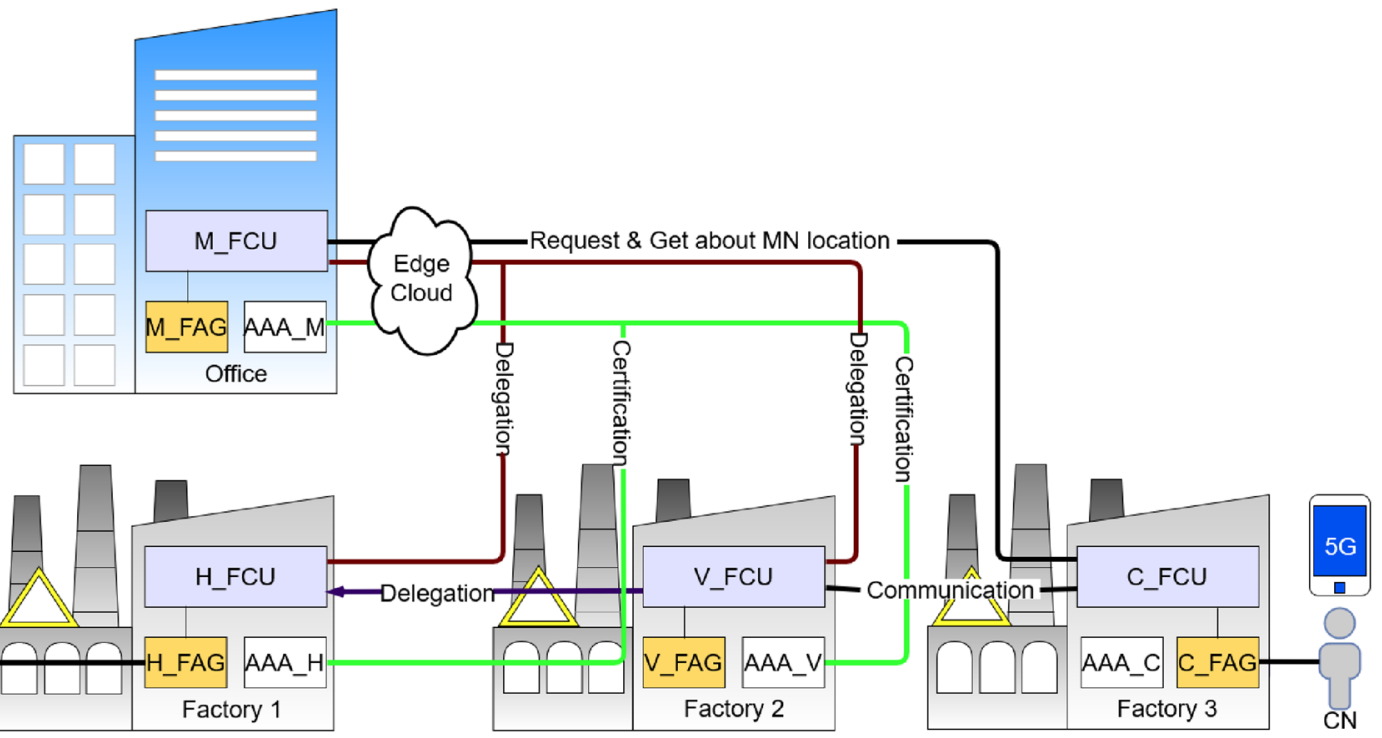

Fig. 4 System architecture

\subsection{Function-distributed mobility management}

In general, the overall network is composed of home network, core network, local network, destiny network, and mobile node. The home network is a network that mobile users register to, while the destiny network is a network that the $\mathrm{CN}$ will access and communicate to. The local network is a network of the MN that is being visited at the moment, and the core network is all the network that generally every data passes through. Figure 5 shows the basic authorization consecutive procedure.

The function of the M_FCU is to authorize location and status information to H_FCU and V_FCU according to the authorization procedure (1), to put the AAA_M certification server in office, and put AAA_H and AAA_V certification server in each factory (2), so that the authorization of certification is carried out between certification servers (3). If the MN moves between each H_FCU and V_FCU in order to save the PMIP environment, they authorize status and location information according to their probability. That is, if the MN moves to the visiting network, the H_FCU authorizes the V_FCU with status, location and the certification-related information. If the $\mathrm{MN}$ enters the visiting network, the V_FAG shoots the quarry to the V_FCU to send the registration request of the MN (4). If the normal registration request is completed (5), the V_FCU sends its changed information to the M_FCU and then improves the environment so that the destiny network can refer to it (6, 7). The V_FCU and M_FCU maintain the connection path information while the status of $\mathrm{MN}$ is maintained. The fail restoring time is calculated with data information of real time information environment. If the total authorization of connection succeeds, the MN communicates with the $\mathrm{CN}$, and receives the best function-distribution service which induces the optimum costs (8).

Figure 6 identifies the additional layer structure. From the perspective of users, the convenient communication layer structure is not different from the seven-layer model for OSI. In fact, the control plan needs the function authorization process. It can accompany the useful path management such as user-based management, which is one of the major requirements for the function distribution mobility. Each distribution function must be automatically maintained and dynamically updated. The management plan adjusts each distribution function management entity.

\subsection{Characteristics of function-distributed mobility management}

In the function distribution mobility, the appropriate adjustment of each function is very important. Table 1 shows the effect not only of the factors of mobility system but also the management technique of each function.

Despite a few exceptions, in general the mobility system factors are dynamically updated. The closer the distribution function is located to where the MN is located, the higher the level of effect. The certification function must be located deep within the internal network. In contrast, the status function must be located at the node that is close to the visiting local network. The path management function may better be located between the source in the middle of the core network and the destination, for the sake of the fast shift of paths. Lastly, the local management function composed of anchoring and casting may be better in the middle place, 
Fig. 5 Basic sequence of delegation procedure

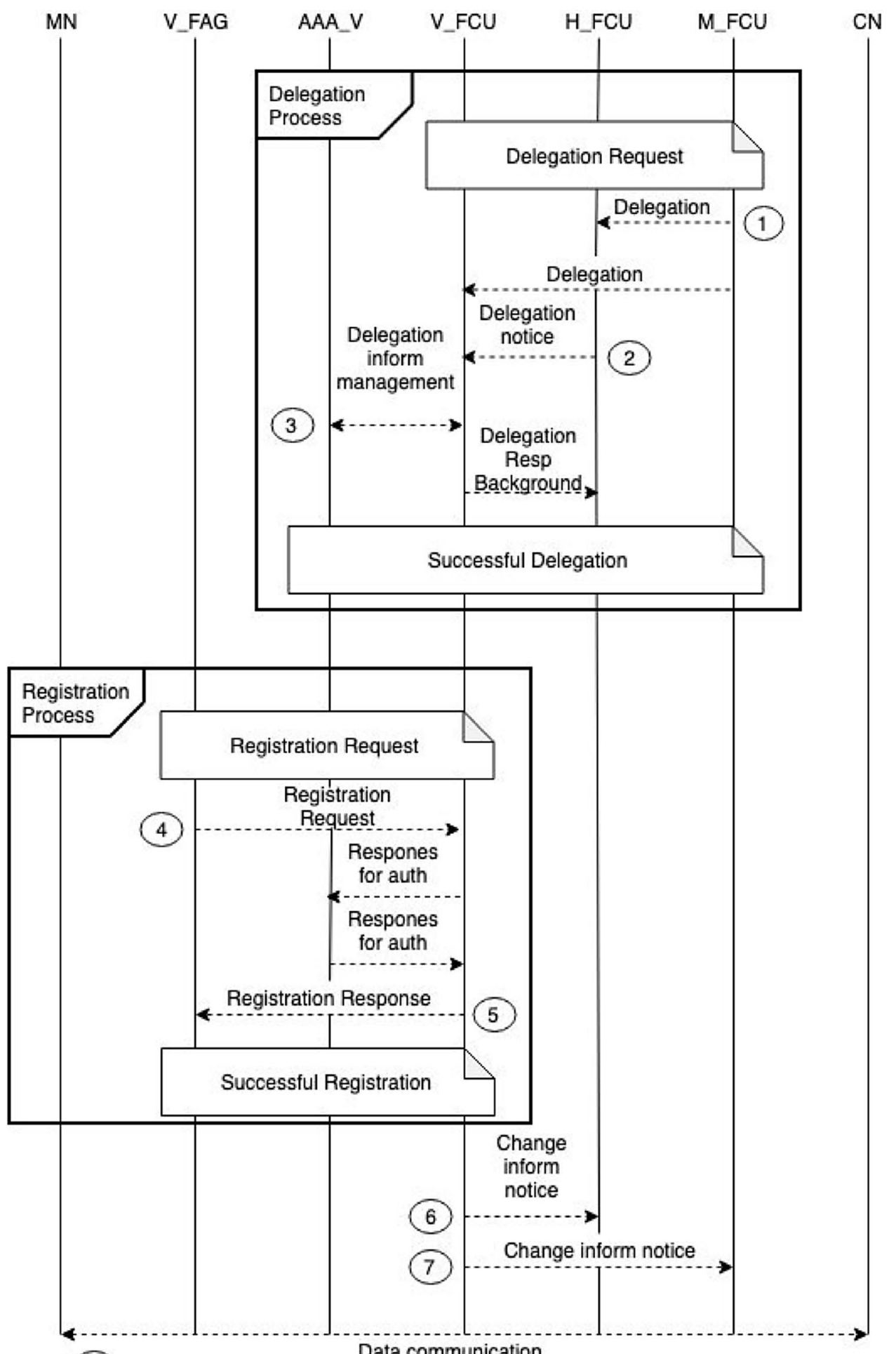

(8)

Data communication like the middle of the local network, which is close to the core network and the MN. Regarding anchoring, the special node provides the status of stable IP address for the NM. In addition, the anchor plays the role of providing low-loss communication when users move. The casting function carries out multi-casting of the user data to the node to which the MN moves beforehand, and attempts to provide. 
Fig. 6 Layered structure of function-distributed mobility
Table 1 Impact of each function placement from mobility system parameter

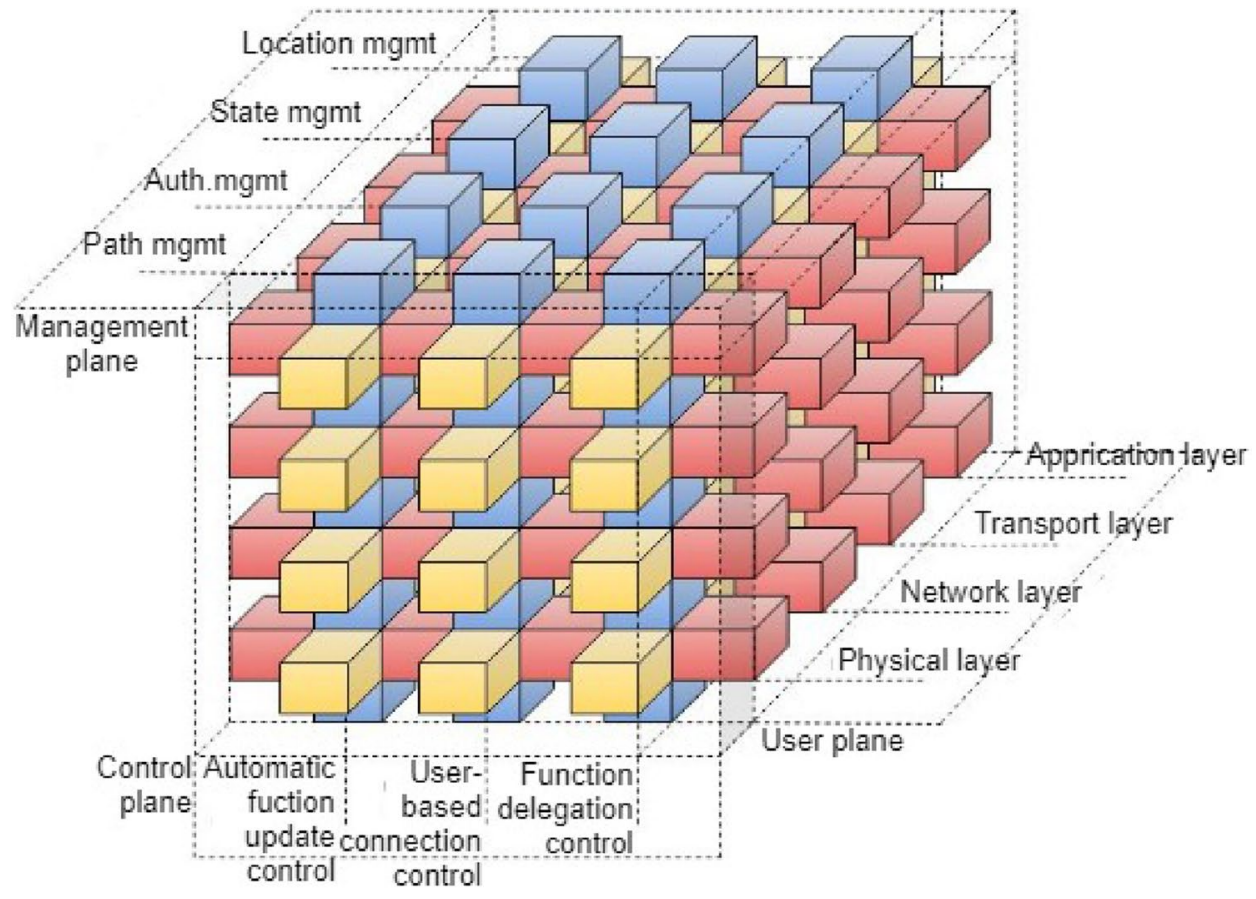

\begin{tabular}{|c|c|c|c|c|c|}
\hline \multirow[t]{2}{*}{ Element } & \multicolumn{5}{|c|}{ Each functional element } \\
\hline & Anchor & Cost & Path & State & Authentication \\
\hline Connection frequency & High & Middle & Middle & High & Low \\
\hline Speed of MN & Middle & High & High & High & Low \\
\hline Recovery time failure & Middle & High & High & High & Low \\
\hline Volume of traffic & Middle & High & High & Low & Low \\
\hline Quantity of MN & Low & Middle & Middle & High & Middle \\
\hline Type of application program & Middle & Low & High & High & Low \\
\hline
\end{tabular}

\subsection{Comparison of function-distributed mobility management and others}

The kinds of differences the function distribution has in comparison with the existing mobility system will be explained. The location update procedure type, anchor point flexibility, function distribution, and path management ability are considered as the comparison target for mobility special measurement.

Table 2 shows the qualitative comparison with other existing mobility methods. From the perspective of the location update type, the MIP always carries out the wide area location update registration. Other methods carry out the local location update function with the wide area location update, and use the cost-effective registration procedure. From the aspect of the anchor point flexibility, the MIP and HMIP use fixed points. In contrast, other environments dynamically change their anchor points according to user priority and environmental condition. The DisMob functions to manage the transmission path from source to destiny. The
Table 2 Qualitative comparison between mobility systems

\begin{tabular}{lllll}
\hline & Location & Anchor & Path & Function \\
\hline MIP & Wide area & Fixation & Fixation & Concentration \\
HMIP & $\begin{array}{c}\text { Wide area \& } \\
\text { region } \\
\text { DisMob }\end{array}$ & Fixation & Fixation & Concentration \\
& $\begin{array}{c}\text { Wide area \& } \\
\text { region }\end{array}$ & Flow & Flow & Dispersion \\
$\begin{array}{c}\text { Proposed } \\
\text { scheme }\end{array}$ & $\begin{array}{c}\text { Wide area \& } \\
\text { region }\end{array}$ & Fixation & Fixation & Dispersion \\
\hline
\end{tabular}

distribution mobility that leads to system construction that is flexible to the function distribution is the only way to satisfy this property. The construction of this function distribution is possible with DisMob and the suggested technique, and is one of the important factors that discriminates it from the existing systems.

Figure 7 shows the envisioned framework, which relies on an SDN-based factory core network, which implements three important building blocks: the data plane, smart factory 
control plane (SFCP) acting as the SF control plane, and NFV. The data plane consists of FCUs that act as SDN switches, and provides the fundamental functionality of packet treatment within the network. This provides the required agility of SF networks, by enabling a rule-based control of individual data flows. The network and related services are managed by the (logically) centralized SFCP. In its role as SDN controller, it is responsible for managing data flows according to changing requirements. The SFCP also orchestrates the services provided by NFV, in terms of deployment, configuration, and monitoring. Furthermore, it provides an abstract management interface to the manufacturing execution system, and implements security policies. The network itself is complemented by NFV. Network functions virtualization enables the SFCP to deploy virtualized services on demand. These services could be network related, but might also be directly associated with the manufacturing process, e.g., in terms of computationally intensive tasks, like image processing. In contrast to a traditional device bound deployment, virtualized services can be instantiated, replaced, or destroyed dynamically, according to changing requirements, e.g., to scale the number of active instances, or change their location within the network topology. While the software-based core network replaces most of the traditional switches and routers, we envision a separation of concerns regarding hard real-time communication and safety. This means that already established and approved technologies, like Ethernet-based field-buses, will continue to be used, especially for scenarios that have stringent safety requirements, and depend on deterministic time synchronization. In the long term, such special purpose domains might be complemented or replaced by software-based manufacturing networks that can provide real-time properties [e.g., as described in Nayak et al. (2015)]. Both technologies can coexist within our framework (Fig. 7). However, other network traffic outside of the special purpose domains is completely under the control of the proposed architecture. For example, this includes the communication between factory floor and manufacturing zone, as well as connectivity towards office IT, or remote production facilities that are connected via wide area network or Internet. These traffic flows are either configured according to actual demands, e.g., to execute an order scheduled by the manufacturing execution system, or pre-configured, in terms of administrative policies. Thereby, the SFCP incorporates different Quality of Service requirements, like bandwidth, latency, or jitter, into its routing and scheduling decisions. It also configures and enforces security policies, by permitting or prohibiting individual data flows inside or across virtual network and security domains. If a fine-grained control of network traffic between domains is desired, virtualized firewalls or deep packet inspection instances are deployed and configured by the SFCP using NFV. These instances screen and filter network traffic originating from possibly untrusted sources. In our scenario, an industrial edge cloud collocated with the production facility provides the resources for virtualized services. Besides the security functionality like firewalls and deep packet inspection mentioned previously, the NFV

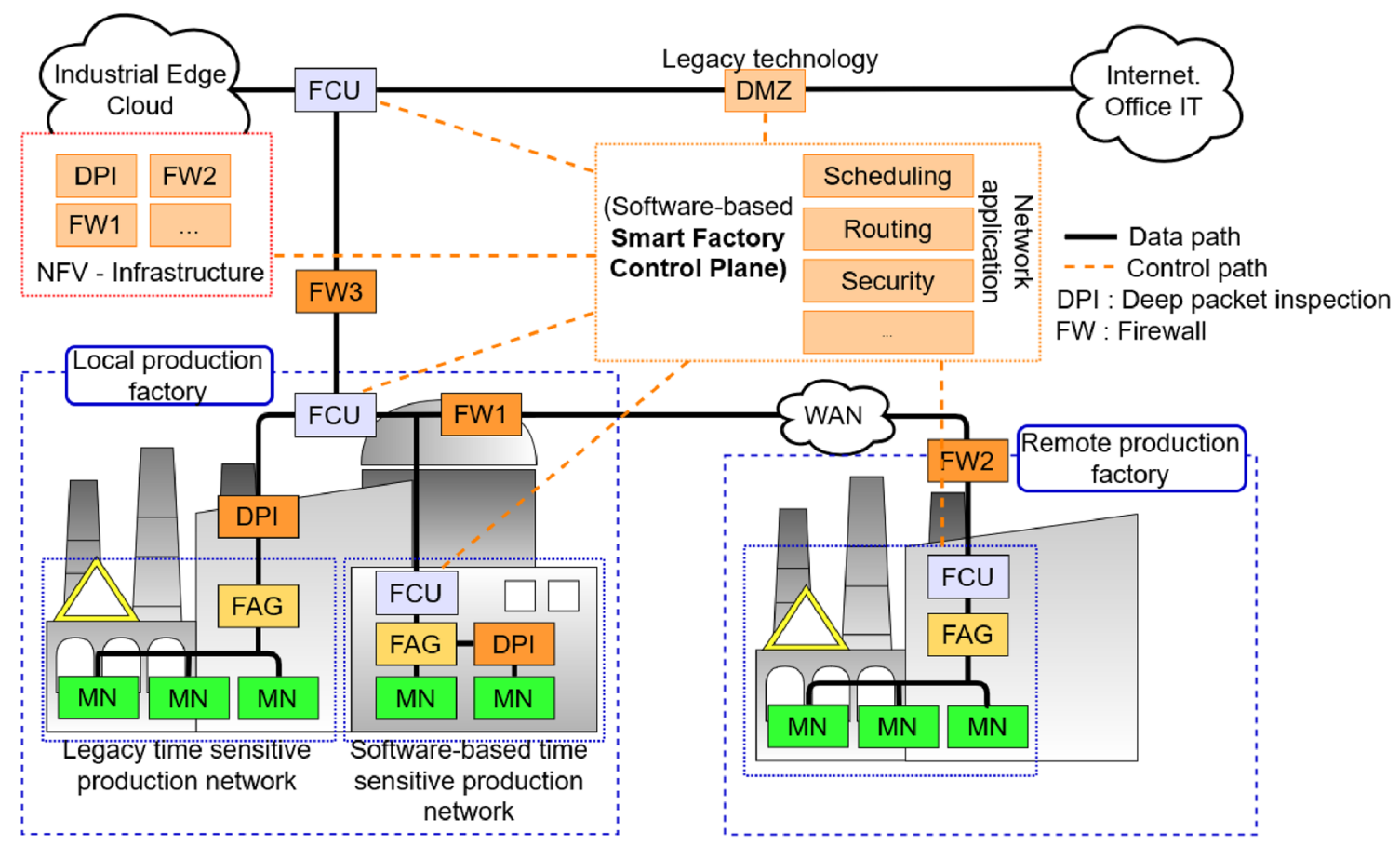

Fig. 7 Architecute for software-based smart factory networking. $D M Z$ demilitarized zone, $L M A$ local mobility anchor, $S D N$ software-define networking, WAN wide area network 
infrastructure can also be used to perform production related tasks, e.g., data processing and filtering.

\section{Performance evaluation}

For analysis of the mobile mobility network, we assume a hexagonal model. The mobile mobility model of hexagonal it is assumed that a FAG consists of the same number of rings in the domain FCU and each ring $r$ is composed of $6 r$ cells. The number of $N(R)$ in the cell reaching the ring $R$ is calculated by the following equation.

$N(R)=\sum_{r=1}^{R} 6 r+1=3 R(R+1)+1$.

\subsection{System modeling}

The next Fig. 8 is a one-dimensional Markov chain model used for analysis.

The number of states respectively corresponds to the number of rings of hexagon portable model. The $q$ is the possibility of remaining in the current cell and $\mathrm{MN}$ moves to another cell with a probability of $1-q$. Therefore, MN located in the cell of ring $r$ can move inward with $p^{+}(r)$ probability and inward with a probability of $P^{-}(r)$.

The number of states respectively corresponds to the number of rings of hexagon portable model. The $q$ is the possibility of remaining in the current cell and $\mathrm{MN}$ moves to another cell with a probability of $1-q$. Therefore, $\mathrm{MN}$ located in the cell of ring $r$ can move inward with $p^{+}(r)$ probability and inward with a probability of $P^{-}(r)$.

$P^{+}(r)=\frac{1}{3}+\frac{1}{6 r}$,

$P^{-}(r)=\frac{1}{3}-\frac{1}{6 r}$.

By using the above probability equations $(2,3)$, we can obtain the following movement probabilities $\alpha_{r, r+1}$ and $\beta_{r, r-1}$ expressions for $\mathrm{MN}$ of ring $r$.

$\alpha_{r, r+1}=1-q($ if $\gamma=0)$,

$\alpha_{r, r+1}=(1-q) P^{+}(r)($ if $1 \leq \gamma \leq R)$,

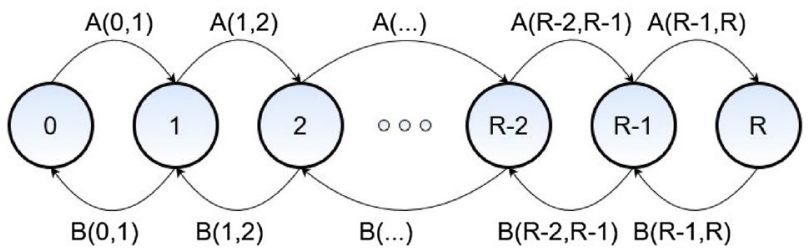

Fig. 8 State diagram for random walk mobility model $\beta_{r, r-1}=(1-q) p^{-}(r)($ if $1 \leq \gamma \leq R)$.

If $\pi_{\gamma, R}$ is defined as the stable state probability of the cell $r$ of the MAP domain with the inner cell of the ring $R$, it can be calculated as follows.

$$
\begin{aligned}
\pi_{r, R}= & \pi_{r, 0} \prod_{i=0}^{r-1} \frac{a_{i, i+1}}{\beta_{i+1, i}}(\text { for } 1 \leq \gamma \leq R), \\
\pi_{0, R}= & \left\{1+\sum_{r=1}^{R} \sum_{j=0}^{\gamma-1} \frac{\alpha_{j, j+1}}{\beta_{j+1, j}}\right\}^{-1} \\
& \left(\text { with } \sum_{r=0}^{R} \pi_{r, R}=1\right) .
\end{aligned}
$$

\subsection{Cost function}

This section defines the cost function considered. In order to evaluate the process that each mobility method possesses and accurate assets. Total cost is analyzed without paging cost. The reason is that paging costs do not always support all methods. So the total cost can be known from the following Eq. (9). $C_{\text {tot }}$ represents total cost, update cost of location information is $C_{l o c}$, and packet transmission cost is $C_{p k t}$.

$C_{t o t}=C_{l o c}+C_{p k t}$.

\subsubsection{Location update cost model}

HMIP, DisMob and the proposed scheme can perform region location update process. MIP executes only the updating process of the wide area position. DisMob and the proposed scheme mean shortening dispersion mobility. Divide the mobility function into several part functions. The function of each part shows the dynamic allocation system to other nodes according to its characteristics. The costs of location update, $C_{H}, C_{L N}$, and $C_{L D}$ are assumed to be the costs of updating locations across wide areas, costs without delegation updates to regional locations, and costs of delegation updates to regional locations.

Execution of wide area location update means that the MN at the boundary of the ring $R$ goes outward. In other situations, the MN performs regional location updates. The $\omega$ arguments for calculating the substitution probability are indicated by $F A G_{V}$ as the probability of executing the delegation process. The probability for performing global location update is calculated in $\pi_{R}, R^{\alpha, R+1}$.

Calculation of the update cost of the position of HMIPv6 follows the following equation.

$C_{l o c}=\frac{\left(1-\pi_{R} \alpha_{R, R+1}\right) C_{g}+\left(1-\pi_{R} \alpha_{R, R+1}\right) C_{l}}{T}$, 


$$
\begin{aligned}
C_{g}= & 2\left[\kappa+\tau\left(D_{v}-D_{c}\right)\right]+N_{C N}+\left(2\left[\kappa+\tau\left(D_{v}-D_{C}\right)\right]\right. \\
& \left.+P C_{C N}\right)+P C_{H A}+P C_{M A P},
\end{aligned}
$$

$C_{l}=2\left(\kappa+\tau D_{v}\right)+P C_{M A P}$.

Calculation of the cost of updating the position of DisMob follows the following equation.

$C_{l o c}=\frac{\pi_{R} \alpha_{R, R+1} C_{H}}{T}+\frac{\left(1-\pi_{R} \alpha_{R, R+1}\right)\left[\omega C_{L D}+(1-\omega) C_{L N}\right]}{T}$,

$$
\begin{aligned}
C_{H}= & 2\left(\kappa+\gamma\left(D_{v}+D_{c}\right)+N_{C N}\left(\left[2 \kappa+\gamma\left(D_{v}+D_{c}\right)\right]\right)\right. \\
& \left.+P C_{C N}\right)+P C_{H A}+P C_{H A i}+P C_{H A D}^{\prime}
\end{aligned}
$$

$C_{L N}=2 \kappa+\tau\left(D_{v}\right)+P C_{L M A_{H}}$,

$$
\begin{aligned}
C_{L D}= & 2\left(Z_{s} Y_{s}+Z_{l} Y_{l}\right)\left[\kappa+\gamma\left(D_{v}\right)\right] \\
& +2 Z_{p} Y_{p} N_{C N}\left[\kappa+\gamma\left(D_{v}+D_{c}\right)\right] \\
& +P C_{H A}{ }^{\prime}+P C_{H A_{i}}{ }^{\prime}+P C_{H A_{i}}^{\prime},
\end{aligned}
$$

The cost of updating the position of the proposed method is calculated based on the following equation.

$$
\begin{aligned}
C_{l o c}= & \frac{\pi_{R} \alpha_{R, R+1} C_{H}}{T}+\frac{\left(1-\pi_{R} \alpha_{R, R+1}\right)\left[\omega C_{L D}+(1-\omega) C_{L N}\right]}{T}, \\
C_{H}= & 2\left(\kappa+\gamma\left(D_{v}\right)+2 N_{C N}\left(\left[\kappa+\gamma\left(D_{v}+D_{c}\right)\right]\right)\right. \\
& +P C_{F C U_{M}}+P C_{F C U_{H}}+P C_{F C U_{v}}+N_{C N} P C_{C N}, \\
C_{L N}= & 2 \kappa+\tau\left(D_{v}\right)+P C_{F C U_{H}} \\
C_{L D}= & 2\left(Z_{s} Y_{s}+Z_{l} Y_{l}\right)\left[\kappa+\gamma\left(D_{v}\right)\right] \\
& +2 Z_{p} Y_{p} N_{C N}\left[\kappa+\gamma\left(D_{v}+D_{c}\right)\right] \\
& +P C_{F C U_{H}}+P C_{F C U_{V}} .
\end{aligned}
$$

$T$ is the average cell registration time, $\kappa$ and $\tau$ are the unit transfer cost on the link with each line and no link. $D_{v}$ and $D_{c}$ are the number of hops between nodes, and $N_{C N}$ means the number of $C N_{s}$ communicating with MN. $P C_{g g}$ is the cost of the process of updating for binding at each node. $*$ refers to the cost incurred in the surrogate process. Finally, $Y_{s / \iota / p}$ and $Z_{s / \iota / p}$ refer to Substitution probability according to each state, location and path management. MIP and HMIP location update cost is calculated in a similar way.

\subsubsection{Packet transmission cost model}

The next is a description of the transfer cost of the packet. Currently to FCU domain $N_{M N}$ means total user. And it is expressed as follows: $N_{F A G}$ indicates the number of FAGs currently connected to the FCU domain. $K$ indicates the average number of users in the FAG range.

$N_{M N}=N_{F A G} K$.

The transmission cost of the packet follows the following equation. $C_{F A G}, C_{F C U}, C_{T}$ means the cost incurred for transmission of packets of FCU and FAG. $C_{T}$ means the transfer cost of a packet between $\mathrm{CN}$ and $\mathrm{MN}$.

$C_{p k t}=C_{F A G}+C_{F C U}+C_{T}$.

$C_{F A G}$ is divided into two parts of lookup and routing cost. The cost of lookup is proportional to the size of the mapping table. The cost of lookup is proportional to the number of MNs. On the other hand, the routing cost is proportional to the log function of the number of FAGs in the FCU domain. $C_{F C U}$ the route optimization is at the node matching the FCU sending the first packet. All packets of thin lines need to be transmitted directly to the MN. $C_{T}$ is the transmission cost. This is related to distance and means the number of hops between MN and CN. Using the previous argument element, the total packet transmission cost can be expressed as follows:

$$
\begin{aligned}
D_{p k t}^{\text {DisMob }}= & \lambda_{s}\left(\left[\alpha N_{M N}+\beta \log \left(N_{F A G}\right)\right]\right. \\
& \left.+\lambda_{s} \theta_{F C U}+\tau \lambda_{s}\left[(S-1)\left(D_{c}+D_{v}\right)\left(D_{c}+D_{h}\right)\right]+\kappa S\right)
\end{aligned}
$$

$$
\begin{aligned}
D_{p k t}^{\text {Proposed }}= & \lambda_{s}\left(\left[\alpha N_{M N}+\beta \log \left(N_{F A G}\right)\right]\right. \\
& \left.+\lambda_{s} \theta_{F C U}+\tau \lambda_{s}\left[(S-1)\left(D_{c}+D_{v}\right)\right]+\kappa S\right) .
\end{aligned}
$$

$\lambda_{s}$ is the session arrival rate, $S$ is the average session size of the packet unit and $\theta_{F C U}$ packet processing cost. $\alpha, \beta$ means an additive element.

\subsection{Results for cost analysis}

Confirm the results based on the mobility model described above. Table 3 shows the numerical values of each element of the system. The evaluation method is analyzed based on MIP, HMIP and DisMob the proposed method.

One of character features of the mobility system is a procedure of updating the position information. The position update cost evaluation is the first. Figures 9 and 10 show the update cost of location along the average cell registration for each of the FCU domain sizes 1, 4. Location update costs are closely related to user mobility. The cell registration time is considered to be a good indicator of the evaluation method. So the location update cost decreases as cell registration 
Table 3 Basic system parameters used in the evaluation

\begin{tabular}{|c|c|c|c|c|c|c|c|}
\hline$P C_{C N}$ & $P C_{L M A}$ & $P C_{L M A}{ }^{*}$ & $P C_{L M A_{i}}{ }^{\prime}$ & $N_{M A G}$ & $N_{C N}$ & $\omega_{M N}$ & $\omega_{M A G}$ \\
\hline 6 & 24 & 24 & 12 & 1 & 2 & 0.1 & 0.2 \\
\hline$Y_{g}$ & $Y_{l}$ & $Y_{v}$ & $D_{h}$ & $\omega$ & $q$ & $K$ & $\alpha$ \\
\hline 0.7 & 0.7 & 0.7 & 6 & 0.55 & 0.4 & 4 & 0.1 \\
\hline$P C_{L M A_{i}}{ }^{\prime \prime}$ & $\theta_{M A G}$ & $\theta_{M A G}^{\prime}$ & $S$ & $Z_{g}$ & $Z_{l}$ & $Z_{v}$ & $\gamma$ \\
\hline 12 & 20 & 20 & 10 & 0.1 & 0.6 & 0.3 & 0.43 \\
\hline$D_{v}$ & $D_{c}$ & $\kappa$ & $\tau$ & $\beta$ & $L_{c}$ & $\lambda_{s}$ & \\
\hline 4 & 2 & 2 & 10.2 & 1 & 20 & 0.1 & \\
\hline
\end{tabular}

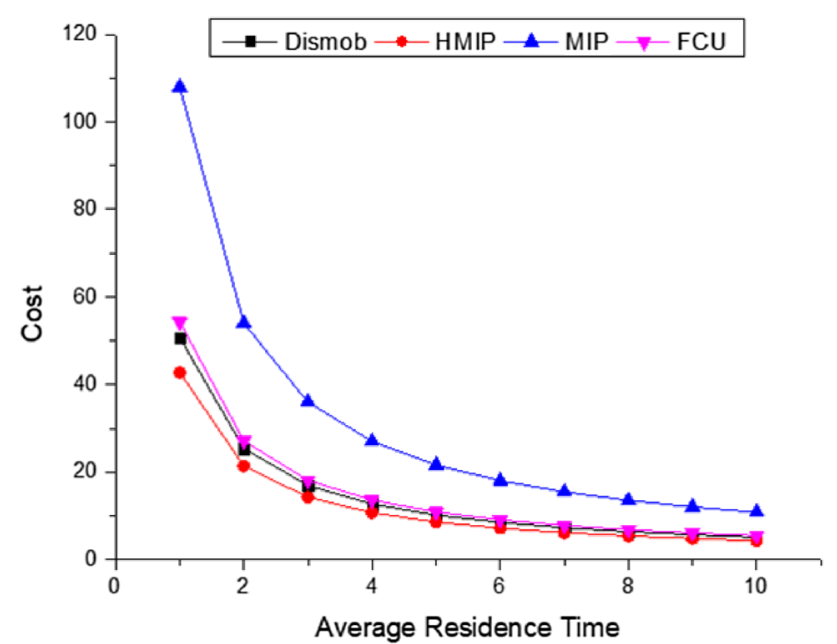

Fig. 9 Location update cost over residence time with domain size 1

time increases. Furthermore, the smaller the R size of the FCU domain, the more frequent it is. The MN executes an update process of a location in a wide area than the location update process of the area. HMIP, DisMob, offering method shows better efficiency than MIP. These three methods omit location renewal costs while $\mathrm{MN}$ is moving in a narrow area for regional location updates. On the other hand, the MIP executes updating of the wide area every time. The FCU domain size R increases, and the MIP cost keeps a constant state. As a result, compared with MIP it is possible to know that the other three methods are good methods from the viewpoint of the cost of updating the position information.

he transmission cost of packets, which is the evaluation of the mobility system has different metrics. In the packet transfer process, the update cost of the location information is related to the user mobility and the number of users involved. In other words, the transfer cost of a packet increases as the number of MNs in the FCU domain increases. MIP is regardless of route optimization, the results are shown in the following Figs. 11 and 12. MIP has the same transfer cost of

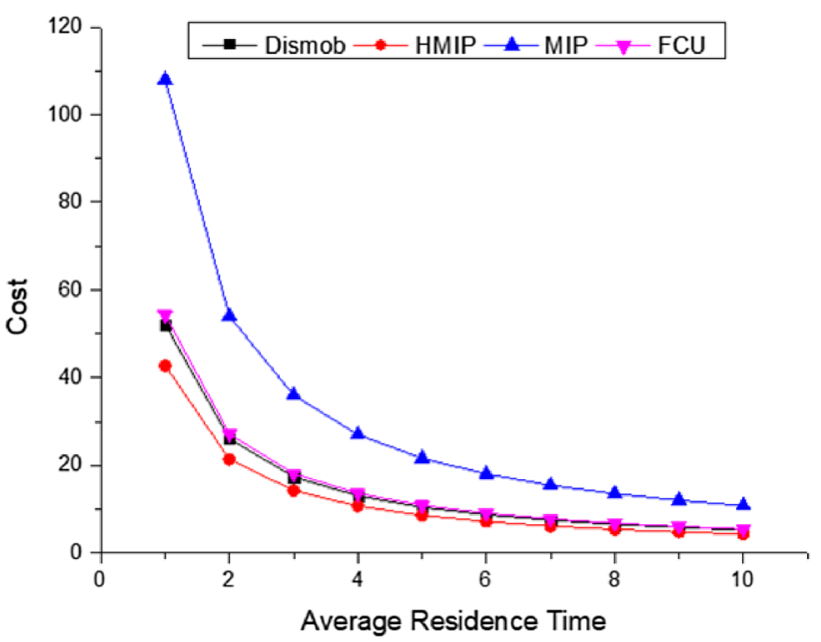

Fig. 10 Location update cost over residence time with domain size 4

packets. The other three methods increase the transmission cost of packets linearly according to the number of MNs. This is because the inquiry procedure cost is linearly related to the number of MNs in the cell. In the case of MIP, it shows the shape of a graph like a constant as the same transport mechanism using MIP tunnel. From the point of view of packet forwarding, it can be seen that MIP is the most effective transmission method compared with other mechanisms.

In the above two methods, I analyzed the position update and packet transmission cost of MIP and the other methods. It is explained the advantages. In analyzing the total cost, we try to provide a more fair assessment by adjusting the elements of delegation. In a system where all elements are taken into consideration, $\gamma$ and $\omega$ are calculated as follows to create the local state.

$$
\begin{aligned}
& 1-\gamma=\frac{\pi R^{2}}{4 \pi R^{2}}=>\gamma=\frac{3}{4}, \\
& 1-\omega=\frac{\pi R^{2}}{4 \pi R^{2}} \Rightarrow>\gamma=\frac{8}{9} .
\end{aligned}
$$




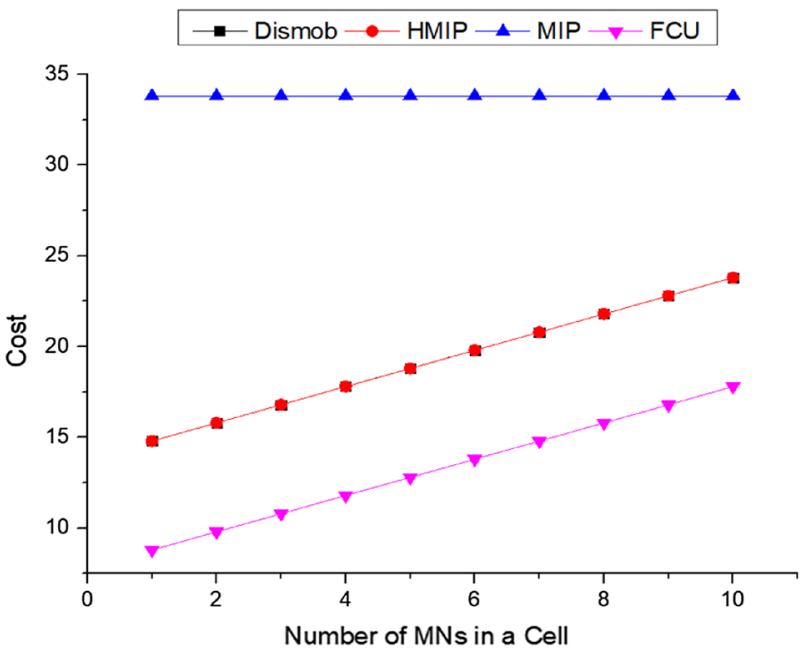

Fig. 11 Packet delivery cost analysis in domain size 1

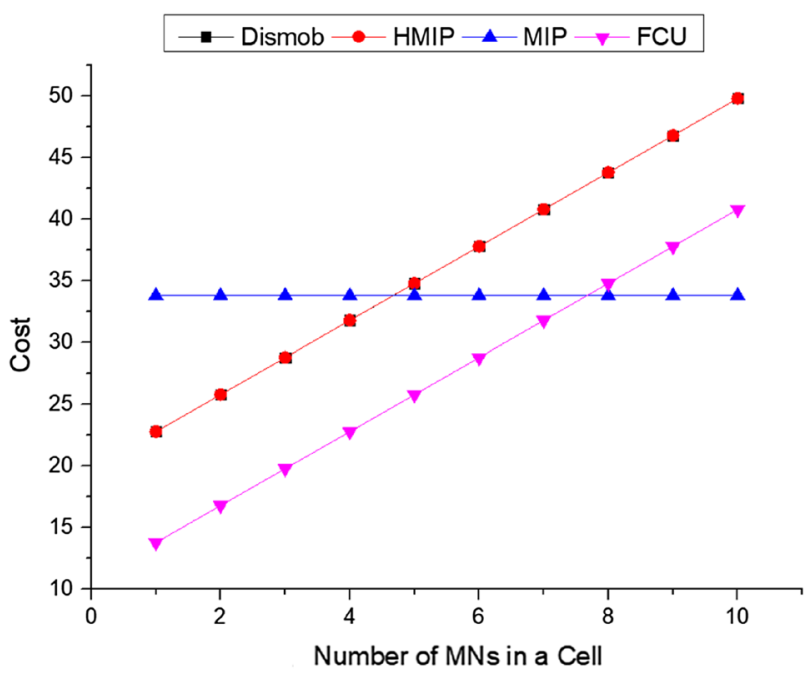

Fig. 12 Packet delivery cost analysis in domain size 4

This can be compared equally in terms of cost analysis when HMIP is $R=3$, DisMob is $R=1$, and the proposed technique is $R=1$. Figures 13 and 14 show the cost of updating the location in the defined area and the transmission cost analysis of the total cost.

The delegation cost is closely related to the update cost of the location information. The transmission cost of the visible packet is closely related to the FCU domain size. In this section, we will explain the analysis of the total cost. For evaluation of liquidity system, session to mobility ratio (SMR) is generally used. In the personal communication service network, SMR stands for complementing cell mobile proportion of mobile network. And it means the relative proportion of session arrival rate with user mobility. The SMR in the Random walk model is defined as follows.

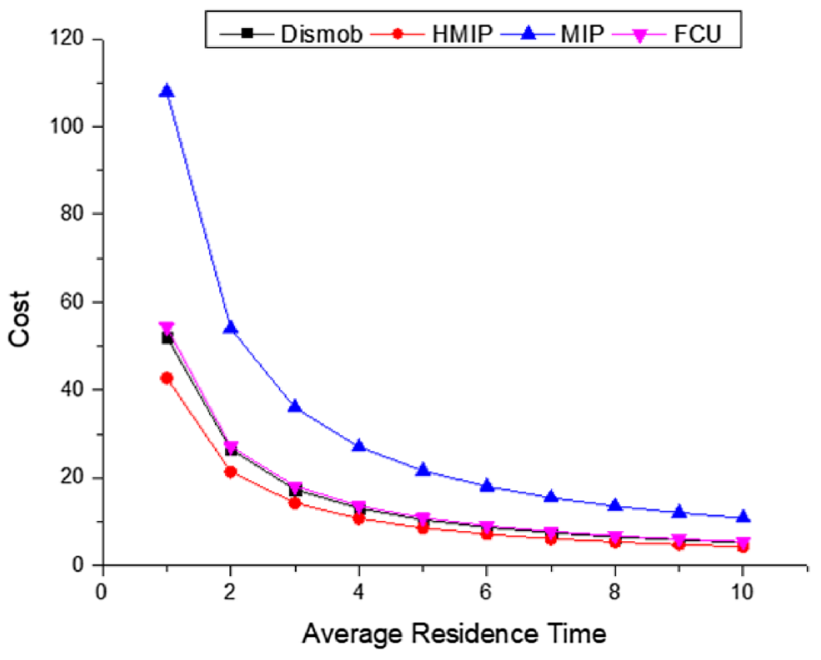

Fig. 13 Location update cost analysis

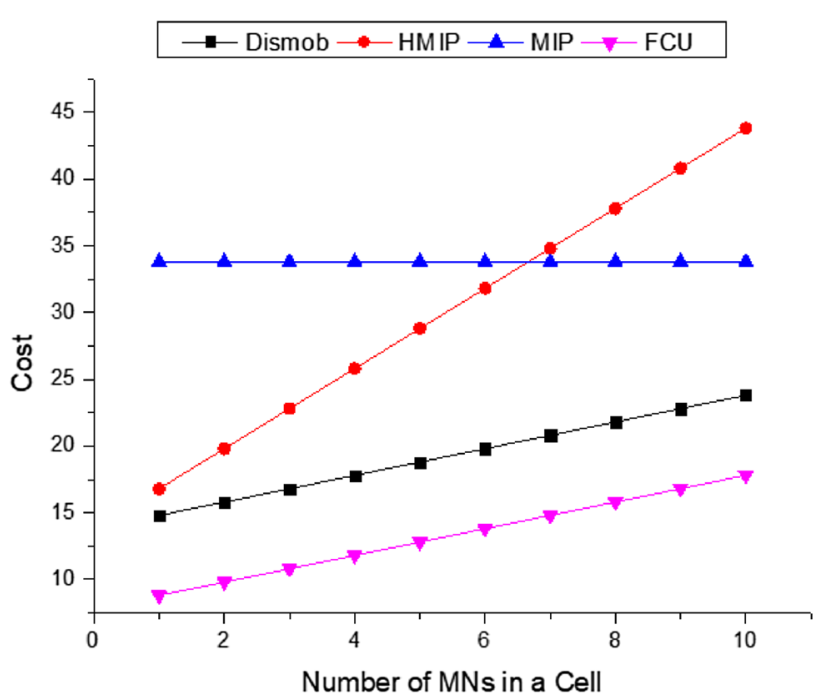

Fig. 14 Packet delivery cost analysis

$S M R=\lambda_{s} T$.

Here, we are trying to make a more fair evaluation by adjusting delegation elements. To make settings for the system where all elements are taken into account, calculate in the following way. By limiting the domain size of HMIP $(R=3)$, DisMob $(R=1)$ and proposed technique $(R=1)$, it becomes possible to evaluate equally in terms of cost analysis.

Figure 15 shows the SMR results for the total area for total cost. If SMR is less than 1, HMIP is better than DisMob and proposed method. However, if SMR is larger than 1 , we can see that the proposed method has good performance. Finally, from the analysis of Figs. 13, 14 and 15, it 
can be seen that the proposed method shows high flexibility through location update cost, packet transmission cost and SMR evaluation.

\subsection{Interoperability for software-based SF networking}

We believe that the concepts of SDN and network virtualization outlined above can help to solve the challenges of future factory networking. As an actively managed infrastructure, software-defined networks can be adopted to the changing demands of SFs. In the following, we present our assumptions regarding key aspects and challenges of SF networks, and discuss the ways in which our software-defined framework addresses these challenges. We envision SFs to be very heterogeneous in terms of machinery, vendors, and equipment types, as well as in terms of data representations, communication protocols, and communication requirements. Industrial networks will become larger and more heterogeneous in terms of both communication requirements and deployed technologies. In addition, the devices themselves will become more heterogeneous, which makes device management especially challenging. Legacy systems will accompany the transformation to SFs for a significant amount of time. We do not expect the large amount of different, often very specialized, automation protocols and standards to converge into a single one soon, if at all. Instead, we assume that future network architectures and frameworks might integrate a wide range of existing solutions. Thus, interoperability with industrial communication architectures and protocols, such as Ethernet based field-buses, or OPC $\mathrm{UA}$ as protocol for machine-to-machine communication, is expected to be an important aspect of SF networks. Considering these assumptions, the challenges that need to be addressed are twofold: on the one hand, SF networks must enable

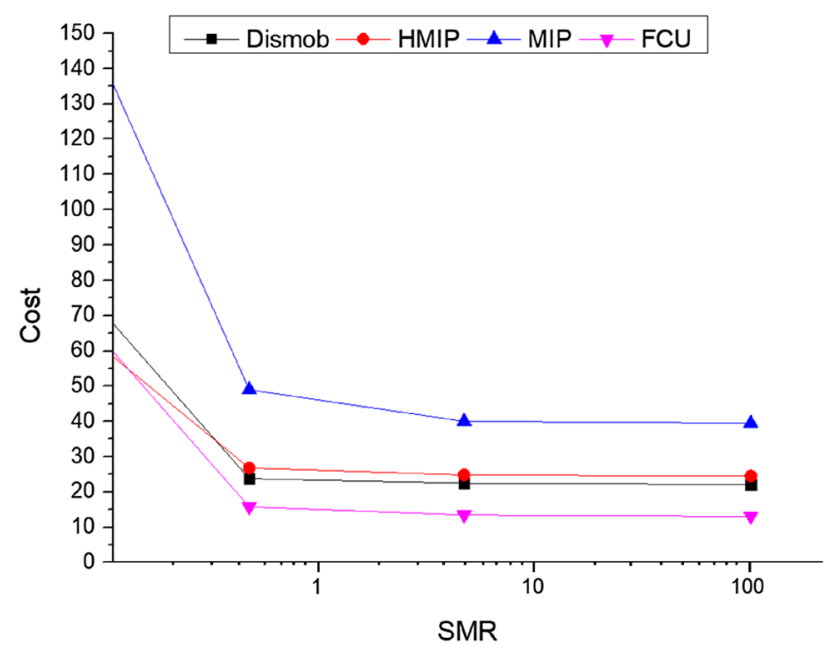

Fig. 15 Cost analysis in unified local area connectivity across a wide range of communication technologies and standards. Thus, the network must both provide the physical hardware to interface with the different deployed technologies, while also acting as an agent between devices using incompatible protocols. On the other hand, device management must also be addressed, to provide an abstracted management interface to the manufacturing execution system. The network itself also requires such facilities, to perform configuration changes during its adaption process. The flexibility of the SDN paradigm enables SF networks to cope with different protocols and standards. The our proposed scheme can be easily extended to support new protocols by means of the software modules provided by different vendors, whereas FCUs with the SDN switch role need to implement approaches like protocol-oblivious forwarding. Virtualized gateways deployed on demand (by using NFV) enable devices using incompatible protocols or standards to communicate with one another. Due to standardized open interfaces for remote programmability, the SDN paradigm provides a foundation for easy to use and efficient automated network management tools. Within a SF, automatic software based mass configuration and management of networking devices is possible, which might be less prone to misconfigurations caused by human failure, or due to the usage of different (vendor specific or communication protocol dependent) management systems. Furthermore, it also makes it easier to integrate devices and systems of different vendors. Because software based networks provide sophisticated tools for traffic engineering (based on the global network view) and fine grained centralized network control by default, the factory should be able to easily cope with such dynamics. Figure 16 shows Concept for interoperability in Software-defined Smart Factory Networking. Each module in the OPC UA-based demonstration factory communicates by a different protocol and can be controlled from the main server via a virtualized gateway under the OPC UA. Integrating these different protocols not only makes it easier to manage, but also enables integrated operation to increase plant efficiency. Virtualized gateways reduce the cost of protocol networks and can adapt to changes in the network.

\section{Conclusion}

In this paper, we describe our vision of a framework for software-based SFs based on current trends in Internet-based networking, like software-defined networks and network virtualization. The framework for SDN in SFs as outlined in this paper can provide sufficient flexibility to deal with the increased production agility anticipated for the factory of the future. Standardized open interfaces and the remote programmability of forwarding devices inside the core network can be used to cope with the trends for increased and more heterogeneous industrial networking. This paper proposes 

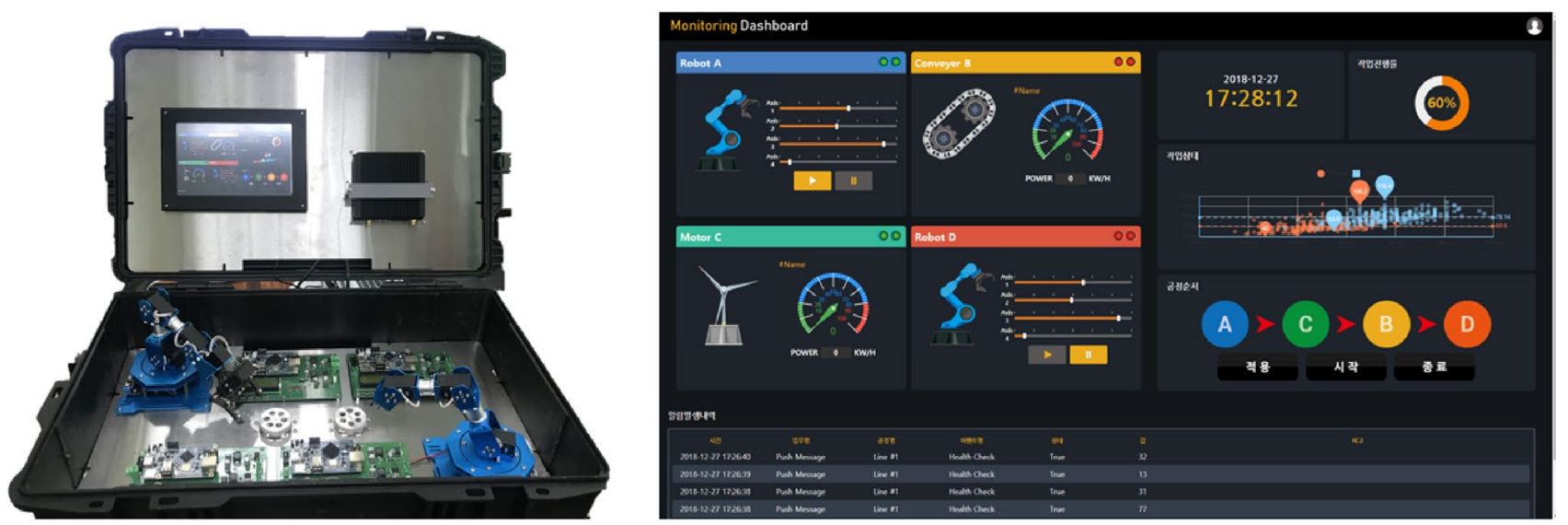

Fig. 16 Proof of concept (PoC) for interoperability in software-defined smart factory networking

the function distribution mobility system, and analyzes the location update cost and packet transmission cost of the existing MIP, HMIP, and DisMob. From the perspective of the mobility method and SMR, the Random-walk mobility model was applied. As the result of cost analysis, the HMIP and DisMob demonstrated similar performances, and better performance than the MIP. At high SMR, the MIP demonstrated better performance, while at low SMR, the other three methods demonstrated better performance. The suggested function distribution mobility method demonstrated better performance not only in the integration region, but also at the cost analysis for the sake of coping with every SMR environment. That is, it has been proven that it is a very effective and flexible method. Therefore, it is confirmed that the proposed method can guarantee global mobility and interoperability in the heterogeneous network environment. The proposed technique supports global mobility from the perspectives of intellectualization and SFs. Therefore, it facilitates cooperation between sites within a factory, and with other factories. It contributes to the establishment of SFs wherein production, networking, and computing are closely integrated. The future research plan is to verify the accuracy of the simulation results through the network simulator and the results of the research through the mathematical analysis, and to study the efficiency of various control messages in the handover between heterogeneous networks.

In the future, $5 \mathrm{G}$ networks will be applied to smart factory environments to efficiently process vast amounts of sensor data. It is believed that efficient systems that have $5 \mathrm{G}$ networks applied to the industrial environment will be able to operate factories efficiently.

Acknowledgements This research was supported by Basic Science Research Program through the National Research Foundation of Korea (NRF) funded by the Ministry of Education (NRF2016R1D1A1B03933828) and the MSIT (Ministry of Science and ICT), Korea, under the ITRC (Information Technology Research
Center) support program (IITP-2019-2018-0-01417) supervised by the IITP (Institute for Information \& communications Technology Promotion).

Open Access This article is distributed under the terms of the Creative Commons Attribution 4.0 International License (http://creativeco mmons.org/licenses/by/4.0/), which permits unrestricted use, distribution, and reproduction in any medium, provided you give appropriate credit to the original author(s) and the source, provide a link to the Creative Commons license, and indicate if changes were made.

\section{References}

Alotaibi M, Lu H, Nayak A (2018) Hierarchical Approach to handle inter-domain mobility in SDN-based networks using mobile IP. In: 5th international cnference on telecom, pp 421-427

Basta A, Kellerer W, Hoffmann M, Morper HJ, Hoffmann K (2014) Applying NFV and SDN to LTE mobile core gateways, the functions placement problem. AllThingsCellular 14:33-38

Bormann C, Castellani AP, Shelby Z (2012) CoAP: an application protocol for billions of tiny internet nodes. IEEE Internet Comput 16(2):66-67

Boulaalam A (2016) Internet of things: new classification model of intelligence. Internation Electron Symp 10:10-19

Chen KT, Su SL, Chang RF (2002) Design and analysis of dynamic mobility tracking in wireless personal communication networks. IEEE Trans Veh Technol 51(3):486-497

Cheng J, Chen W, Tao F, Lin CL (2018) Industrial IoT in 5G environment towards smart manufacturing. J Ind Inf Integr 10:10-19

Choi WJ, Tekinay S (2002) An adaptive location registration scheme with dynamic mobility classification. In: IEEE International Conference on Communications (ICC'02), vol 1, pp 440-444

Condoluci M, Mahmoodi T (2018) Softwarization and virtualization in $5 \mathrm{G}$ mobile networks. benefits, trends and challenges. Comput Netw 146:65-84

Copel R, Crespi N (2011) Policies to enable serving untrusted services on alternative (Non-3GPP) and untrusted access networks in EPS. In: 2011 IEEE 35th annual computer software and applications conference workshops, vol 146, pp 48-53 
Darwish A, Hassanien AE (2018) Cyber physical systems design, methodology, and integration: the current status and future outlook. J Ambient Intell Hum Comput 9(15):1541-1556

Eliasson J, Delsing J, Derhamy H, Salcic Z, Wang K (2015) Towards industrial internet of things: an efficient and interoperable communication framework. In: 2015 IEEE International conference on industrial technology (ICIT), pp 2198-2204

Foundation ON (2013) SDN architecture overview, 1st edn. https:// www.opennetworking.org/

Frias Z, Martínez JP (2018) 5G networks: will technology and policy collide? Telecommun Policy 42(8):619-621

Gandhi R, Liu HH, Hu YC, Lu G, Padhye J, Yuan L, Zhang M (2014) Duet: cloud scale load balancing with hardware and software. In: Proceedings of the 2014 ACM conference on SIGCOMM, vol 44 issue 4, pp 27-38

Ghosh S, Ghosh SK (2019) Traj-cloud: a trajectory cloud for enabling efficient mobility services. In: 11th international conference on communication systems \& networks, pp 765-770

Girbea A, Suciu C, Nechifor S, Sisak F (2014) Design and implementation of a service-oriented architecture for the optimization of industrial applications. IEEE Trans Ind Inf 10(1):185-196

Gundavelli S, Leung K, Devarapalli V, Chowdhury K, Patil B (2018) A comprehensive survey of the current trends and extensions for the proxy mobile IPv6 protocol. IEEE Syst J 12(1):1065-1082

Hong CY, Kandula S, Mahajan R, Zhang M, Gill V, Nanduri M, Wattenhofer R (2013) Achieving high utilization with software-driven WAN. ACM SIGCOMM Comput Commun Rev 43(4):15-26

Hu F, Hao Q, Bao K (2014) A survey on software-defined network and openflow: from concept to implementation. IEEE Commun Surv Tutor 16(4):2181-2206

Jain S, Kumar A, Mandal S (2013) B4: experience with a globallydeployed software defined WAN. ACM SIGCOMM Comput Commun Rev 43(4):3-13

Jin H, Jin Y, Lu H, Zhan C, Peng M (2010) NFV and SFC: a case study of optimization for virtual mobility management. IEEE J Sel Areas Commun 28(8):1344-1362

Johnson D, Perkins C, Arkko J (2004) Mobility support in IPv6. IETF RFC 3775. https://www.rfc-editor.org/rfc/rfc3775

Konieczek B, Rethfeldt M, Golatowski F, Timmermann D (2016) Software-based smart factory networking. Automatisierungstechnik (ICIT) 64(9):765-773

Koodli R (2009) Mobile IPv6 fast handovers. IETF RFC 5568. https:// www.rfc-editor.org/info/rfc5568

Kreutz D, Ramos FMV, Veríssimo PE, Rothenberg CE, Azodolmolky S, Uhlig S (2015) Software-defined networking: a comprehensive survey. Proc IEEE 103(1):14-76
Magnano A, Fei X, Boukerche A (2015) Predictive mobile IP handover for vehicular networks. In: 2015 IEEE 40th conference on local computer networks (LCN), pp 338-346

Modares H, Moravejosharieh A, Lloret J, Salleh RB (2016) A survey on proxy mobile IPv6 handover. IEEE Syst J 10(1):208-217

Moritz G, Golatowski F, Lerche C, Timmermann D (2013) Beyond 6LoWPAN: web services in wireless sensor networks. IEEE Trans Ind Inf 4:1795-1805

Nayak NG, Dürr F, Rothermel K (2015) Software-defined environment for reconfigurable manufacturing systems. In: Proceedings of the 5th international conference on internet of things (IoT 2015), vol 6, pp 122-129

Oughton EJ, Frias Z (2017) The cost, coverage and rollout implications of 5G infrastructure in Britain. Telecommun Policy 2017:1-17

Pan J, SPaul, Jain R, Bowman M (2008) Milsa: a mobility and multihoming supporting identifier locator split architecture for naming in the next generation internet. In: Proc of ieee global communications conference (GLOBECOM'08), pp 1-6

Prinz F, Schoeffler M, Lechler A, Verl A (2018) Dynamic real-time orchestration of I4.0 components based on time-sensitive networking. Procedia CIRP 72:910-915

Rong W, Vanan GT, Phillips M (2016) The internet of things (IoT) and transformation of the smart factory. Internation Electron Symp 10:10-19

Santoyo-González A, Cervelló-Pastor C (2018) Latency-aware cost optimization of the service infrastructure placement in $5 \mathrm{G}$ networks. J Netw Comput Appl 114:29-37

Sezer S, Scott-Hayward S, Couhan PK, Fraser B (2013) Are we ready for SDN? Implementation challenges for software-defined networks. IEEE Commu Mag 64(7):36-43

Soliman H, Castelluccia C, ElMalki K, Bellier L (2008) Hierarchical mobile IPv6 (HMIPv6) mobility management. IETF RFC 5380. https://www.rfc-editor.org/info/rfc5380

Zheng R, Ge Y, Hou JC, Thuel SR (2002) A case for mobility support with temporary home agents. ACM SIGMOBILE Mob Comput Commun Rev 6:32-46

Publisher's Note Springer Nature remains neutral with regard to jurisdictional claims in published maps and institutional affiliations. 